P. Altmeyer, K. Hoffmann, M. Stucker (eds)

\section{Skin Cancer and UV Radiation}

Springer Verlag, Berlin 1997

1331 pp.; USD 225.00

ISBN 3-540-62723-5

UV radiation has been known to be a major risk factor in the development of skin cancers. A conference was held in Bochum, Germany, in 1996 on this subject, bringing together all the leading world experts. The proceedings of this conference are now available in published form, dealing with physiology, biochemistry, molecular biology, prevention, detection and treatment. The book contains over 1390 pages and 163 contributions from as many authors. The material is organized into broad subject matters. The individual contributions deal also with national health policies on sunlight avoidance. A great deal of information is presented on sunblockers, including their physics and effectiveness. Several chapters deal with unusual case reports and treatment methods. Melanoma and its treatment is discussed. Particularly interesting are the chapters on skin cancers and UV radiation in the history of medicine.

This book is obviously important as a historical record of the conference and as a collection of a variety of information on the subject. It is not synthesized in any way, but presented as the speakers presumably articulated their data. The book is of little use to those with a more casual interest in the area. However, it is an important addition to a major medical library's collection. Researchers in this area will want to know more details as written in the primary journal publications. The public health issues are also important to document. An important contribution to the oncology field is the understanding that sun blockers do not prevent melanoma.

Paul P. Carbone, Madison, Wisc.

Beverly A. Teicher (ed.)

\section{Antiangiogenic Agents in Cancer Therapy}

Humana Press, Totowa, 1998

USD 145.00

ISBN 0-896-03641-3

The latest book in a series on Cancer Drug Discovery and Development is edited by Beverly Teicher and is entitled Antiangiogenic Agents in Cancer Therapy. This book focuses on understanding how tumors get their blood supply, the biology of tumor dissemination as it relates to angiogenesis and the early development of treatments designed to interfere with the angiogenesis-related metastases. The book consists of 25 chapters divided into 3 sections. The first deals with angiogenic factors and tumor vasculature. The second deals with antiangiogenic factors and the third with in vivo studies and clinical trials. The chapters are authored by well-known experts and well referenced. This field has moved slowly for many years, but now there is an explosion of knowledge that has moved quickly into clinical studies. While the early clinical trials have not proven to be spectacularly successful, they do offer some hope for the future.

The book has a great deal of background material. Important is the understanding of how many diverse factors interact with each other. Studies of blood flow, angiogenesis, hypoxia and cellular $\mathrm{pH}$ that in the past appeared to be unrelated now have been shown to impact on the field. These observations now seem to be closely tied into the microenvironment of organs, numerous growth factors and the process of neoangiogenesis. The first section represents a truly extensive synthesis of many years of work.

The second segment deals with the antiangiogenesis agents themselves. Individual chapters address 7 different classes of angiogenesis agents. These include squaline, shark cartilage, thrombospondin, matrix metalloproteinases, angiostatin, thalidomide and dietary and nutritional modulation of tumor angiogenesis. Again, these chapters are well referenced and contain excellent summaries of the current state of knowledge.

The last section contains 9 chapters that involve mainly the experimental in vivo data and early clinical trials of the agents. In addition, 2 chapters deal with how one evaluates these agents clinically. It is clear that the standard cytotoxic phase I trial methodology will not be sufficient. These agents are not myelotoxic, and escalation of the doses to toxicity is not likely to be rewarding. Defining what the dose should be will require elucidating intermediate endpoints that will need validation by large-scale trials. Moreover, performing these trials in advanced heavily pretreated metastatic cancer patients may not reveal the biologic endpoints that one is looking for. Tumor regression, a standard endpoint in cytotoxic trials, may not be a reasonable endpoint. Another chapter in this section deals with the prognostic value of tumor vascularity and difficulties with quantifying the vascularity. One disappointment is that there is no synthesis and summary of the field. The future of how this area is moving is not presented. While some chapters have summaries, other do not.

This field has moved very slowly. The reasons are clear. It is very complex and involves many components. The biology of angiogenesis has many activating factors and a host of proteins and enzymes that counteract the process. Angiogenesis is obviously very important to normal tissue growth and repair as well as the inflammatory and immune responses. Tumors also regulate angiogenesis and vascularity. They seem to be able to survive under very adverse conditions.

\section{KARGER}

Fax + 41613061234

E-Mail karger@karger.ch

www.karger.com
(C) 1999 S. Karger AG, Basel

Accessible online at:

http://BioMedNet.com/karger 
They also appear to be able to turn off antiangiogenesis factors that would restrict tumor growth. All of this adds up to the fact that the ability to use these agents clinically to inhibit tumor vessel growth will be difficult. The problem of creating agents that specifically inhibit tumor vessel growth and not normal cells is challenging. Likewise, one needs to get these agents into tumors (localization). Finally the tumors may have ways of inactivating these factors that need to be understood and overcome. The clinical trials to develop these agents are not clearly defined. Neither is the population of cancer patients that would benefit from these agents.

Overall, this book makes an important contribution and has a place in research libraries of both academia and industry. It represents a current detailed analysis of the various components of the biology of antiangiogenesis field. The editor seems to have encouraged the rapid publication of the material. The references include some very recent papers. However, the field is moving quickly and the material will need to be updated. Hopefully this will occur.

David P. Carbone, Nashville, Tenn. Paul P. Carbone, Madison, Wisc.

\section{Andrea Manni (ed.)}

\section{Endocrinology of Breast Cancer}

Humana Press, Totowa 1998

400 pp.; USD 125.00

ISBN 0-896-03591-3

Endocrinology of Breast Cancer is the 20th volume in a series on Contemporary Endocrinology (series editor, P.M. Colin). This latest volume edited by A. Manni contains 23 chapters by 41 international contributors. The chapters are written to be stand-alone pieces, although a great deal of the material is interrelated. This occurs because the book is organized into 6 sections that deal with benign breast disease, mammary development, carcinogenesis, tumor progression, targets for treatment and endocrine therapy. Clearly, hormones and oncogenes, growth factors and receptors interact at many points.

Hormones play an important role in the development of the normal breast as well as breast cancer. More than 100 years ago, Beatson described the beneficial effects of oophorectomy in women with advanced breast cancer. Earlier in this century, surgical ablative procedures were the only means of palliative therapy. Then came the use of hormones such as estrogens, androgens and progestins. Since the 1970 s, specific hormonal agents have been developed that have replaced the ablative procedures. The discoveries of hormone receptors and tamoxifen have further enhanced our ability to interfere with breast cancer growth. These discoveries not only began to define who should be treated, but also have helped us gain insights as to how hormones work. In the past two decades, knowledge of genes and oncogenes has increased rapidly. Now the picture become clearer as well as more complex. The table in Chapter 14 by Klijn and colleagues lists 34 different factors that affect breast cancer prognosis. Obviously, many of these factors are interdependent. Knowledge will be the beacon with which we solve the complex puzzle as we strive to learn more about earlier diagnosis, more effective treatments and even prevention of breast cancer.
The book is aimed at clinical specialists as well as experimentalists. It is not overly dense, and the material covers background information such as interesting historical references to breast cancer as well as new data. There are relatively few illustrations, charts and tables; all in black and white. The references are recent. I strongly recommend its use by those involved in breast cancer treatment. It is one of the few books that deal with the management of benign breast conditions.

Paul P. Carbone, Madison, Wisc.

Jimmie Holland

\section{Psycho-oncology}

Oxford University Press, Oxford 1998

118 pp., USD 139.50

ISBN 0-19-510614-8

Psycho-oncology addresses the 'psychological, social, and behavioral dimensions of cancer from the patients' perspective and their families and how these factors influence morbidity and mortality'. The volume is intended to provide the 'reader with a base to understand and treat patients and to counsel healthy people' at risk for developing cancer. Dr. Holland has gathered a host of collaborators to write more than 102 chapters. The chapters cover psychological and behavioral factors, cancer risks, adaptation to cancer, treatment and specific cancer sites. In addition, they cover psychiatric disorders and their treatments, childhood cancers, family and staff support issues. The final chapters discuss ethical, research international and policy issues. The book is truly comprehensive. There is little that is missing.

The book serves as an important addition to the literature for those who practice in this area, but can serve as an important reference to others. The material will give them a good basic data set on cancer biology, treatment and the various cancer sites. For medical oncologists, the book provides an excellent resource, providing a comprehensive resource to learn new facts that will help them gain insight in understanding the reactions of cancer patients and their families. To those oncologists who are interested in preventing cancers, this book provides insights into behavior barriers. The material also covers issues of underserved populations, the elderly and children. In addition, there are chapters on staff support and training in psycho-oncology. All too often we do not recognize or know how to treat the stress and psychological difficulties faced by staff taking care of patients. Dr. Holland also describes how cancer centers can establish psycho-oncology units.

The chapters are well written with a short summary that helps those who want to understand the bottom line. The breadth of each chapter makes them stand alone as a resource. The references are detailed and provide additional material to look at.

This book is an important addition for all libraries at cancer centers. It will become the reference resource for this oncology specialty for years to come.

Paul P. Carbone, Madison, Wisc. 
H.M. Pinedo, G. Giaccone (eds)

\section{Drug Resistance in the Treatment of Cancer}

Cambridge University Press, Cambridge 1998

340 pp.; USD 95.00

ISBN 0-521-47321-7

This book is a collection of chapters by various authors reviewing various topics related to drug resistance in cancer. As with most compilation books, it has the strengths and weaknesses of multiple different authors, writing styles and topics which often do not blend together very well. It would also appear that the editors suffered the malady common to these endeavours, lack of timeliness. Some of the sections are reasonably up-to-date with references from 1996 and 1997. Other sections are clearly not that recent.
The section on alkylating agent resistance is almost a book in itself. It starts out very thoroughly, updating the reader on the many potential areas of study pertaining to the topic. But then, the chapter meanders through areas with a diffuse relationship to alkylating agents. It appeared, the authors wrote their section and then added to it $1-2$ years later.

The strength of the book is related to the weaknesses described above. Multiple knowledgeable authors review topics related to drug resistance. Some of the authors primarily review their own work, while others try and update the reader on significant contributions from others.

The book accomplishes what it is supposed to; it provides a way for the reader to learn and somewhat update his/her knowledge of drug resistance.

Howard Bailey, Madison, Wisc.

IARC Handbooks of Cancer Prevention

Vol. 2: Carotenoids 1998

$326 \mathrm{pp}$, £ 45.00, Oxford University Press

ISBN 92-832-3002-7

\section{Cancer Incidence in Five Continents Vol. VII}

D.M. Parkin, S.L. Whelan, F. Ferlay, L. Raymond, J. Ferlay (eds.)

1240 pp, \$ 129.00, Oxford University Press

ISBN 92-832-2143-5 